\title{
Teaching Astronomy at Sydney Observatory
}

\author{
By N.R. Lomb \& J. Kitchener \\ Sydney Observatory, PO Box K346, Haymarket 2000, Australia
}

\section{Introduction}

Sydney Observatory is a museum of astronomy and a public observatory. It is Australia's oldest existing observatory and is now a branch of the Powerhouse Museum, the largest museum in the southern hemisphere. With 65,000 visitors each year, the observatory is popular with the public. Visitors can come during the day to see exhibits and audiovisuals and in the evenings on telescope viewing sessions. They can also take part in school holiday workshops, adult education courses or a telescope-making course. In addition, many school groups come along during the school terms to extend the astronomical knowledge of their students. Other professional services provided by the observatory include an annual guidebook with up-to-date information for the sky as seen from Sydney and an astronomical information service for the public and the media.

In this paper we will mainly discuss selected aspects of our educational activities, exhibitions and equipment, highlighting recent developments in the 1990s.

\section{Recent Innovations in Education}

\subsection{Open Nights}

A maximum of only 45 people can be accommodated at any one time in one of our regular evening sessions. During school holidays this is nowhere near enough to meet the demand. When there is a major astronomical event, we like to give more people a chance to look through our telescopes. At these times we organise open nights at which up to 1000 people can attend.

Special open nights have been held to view lunar eclipses, a favourable opposition of Mars and the ring plane passage of Saturn. During the collision of Comet ShoemakerLevy 9 with Jupiter we held six open nights, each attracting over 1000 people. A recent open night targeted Comet Hyakutake. For this event we chose a Saturday night just before the comet disappeared from view in the southern hemisphere. Although viewing could only start at midnight, about 500 visitors attended. As these events attract much media interest, the open nights receive much free publicity.

Open nights are also held on less rare events. In the school holiday time when the observatory is very busy, a particular planet or constellation is targeted. For example, the target could be Jupiter, Saturn, or the Southern Cross.

On an open night the two telescopes in the domes are used as well as telescopes in the grounds. These include a 14 in $(35 \mathrm{~cm})$ Celestron Schmidt- Cassegrain which has a fixed pier in the grounds of the observatory and three smaller portable telescopes. In addition, we encourage people who have just completed a telescope-making course at the observatory to bring along their finished telescopes. Talks with up-to-date images are held at regular intervals, continuous videos are shown and the exhibitions are open. Refreshments are provided by school students raising money for their school. Numerous guide lecturers are available to assist the visitors.

Open nights give a wonderful learning opportunity to many people who otherwise may not visit the observatory. By not having to book there is no prior commitment and the participants can make a decision to attend on the night. 


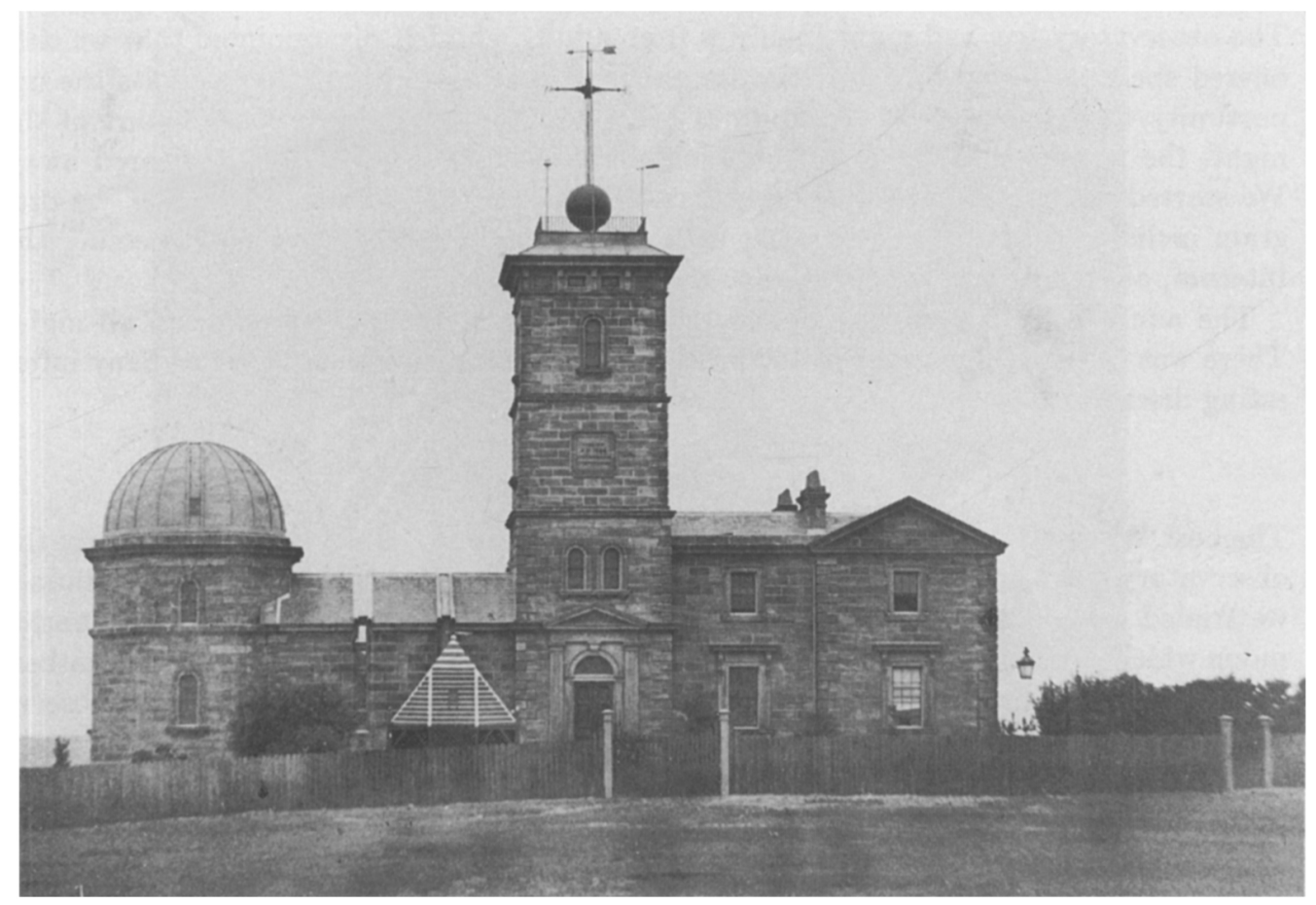

Figure 1. Sydney Observatory in the 1860 s.

\subsection{Camp-ins for Children}

These are held for secondary school students (approximately $12-17$ years of age). Generally, there are two held in each year, one in the winter school holidays and one in the summer holidays. They are very popular and there is usually a waiting list for them. Twenty students attend each camp-in at a time.

If the night is clear, there are several viewing sessions held throughout the night interspersed with other activities. The format of the camp-in can vary but ordinarily it includes a planisphere-making workshop, a demonstration of our computer programs and finding astronomical information using the Internet. On a cloudy night an astronomical quiz and a session in our tiny planetarium are included. Our use of computer programs and the Internet is discussed in more detail in Lomb, 1996.

The students at the observatory are well provided with nourishment: dinner, supper and breakfast are served and extra food and drink are available on demand. The students are asked to bring warm clothes and sleeping bags, though understandably many do not get around to sleeping during the night. In the early hours of the morning the computerised Meade telescope (section 3.2) is available for their use. The students can choose objects that they want to view from a catalogue and then find them using the telescope. They can also rest or watch pseudo-science videos.

The camp-ins are a wonderful learning experience as they provide enthusiastic youngsters with an opportunity to feel at home in the impressive environment of an observatory. They are constantly being stimulated and the activities are varied. There is also a lot of informal time when the students and our specialist staff can interact. 


\subsection{Camp-ins for Adults}

The observatory has had many inquiries from adults who felt disappointed that we only offered specialised and fun activities for children. Last summer we gave adults the opportunity to stay overnight. In spite of exceptionally bad weather at the start of the night, the response was tremendous. Fortunately, the clouds eventually cleared away. We started later than with the youngsters and offered supper and breakfast. The program included a planisphere-making workshop, computer programs and accessing the Internet, as well as viewing through to daylight.

The adult camp-in was a great success with most participants staying up all night. There was plenty of opportunity for informal interaction with the staff and many interesting discussions ensued.

\subsection{Midnight Suppers}

The best viewing hours are after the normal closing time of the observatory. To make the observatory more accessible and to give the viewing a more sophisticated feel in summer we trialed midnight viewings with a champagne supper. We targeted the last quarter moon which can never be seen at our regular sessions. Again, despite initial bad weather, there was an excellent response from the public. Later the sky cleared enough to observe the moon.

These midnight suppers have been repeated, sometimes with a special theme such as the Rebirth of the Sun held on our winter solstice in June. This particular event attracted 65 participants including a group of new age people who specialise in reciting chants to nature.

Midnight viewing attracts people who want exciting experiences with a difference. It gives an excellent exposure to astronomy for an audience who would not normally come to a regular viewing session.

\section{Viewing the sky}

\subsection{Light pollution}

For members of the public the highlight of an evening visit to Sydney Observatory is the chance to look through a large telescope. Although the observatory is in the centre of a large, light polluted city, the planets and many of the signposts of the southern sky are still visible. These include the double star Alpha Centauri, the globular cluster Omega Centauri and the famous open cluster Kappa Crucis.

Recently there have been some threats of further increase in sky brightness. One of these was from the floodlighting installed to illuminate a nearby office building. Each of its four 125 metre high concrete columns was lit from below by six powerful lights aimed almost directly upwards. The high reflectivity of the concrete meant that beams of light, extending high above the building, were reflected off the concrete columns.

We protested to the local city council who had authorised the installation and we were supported by an amateur astronomy group who has a long association with Sydney Observatory. The amateur astronomers organised a petition of over one thousand signatures. As well, we have tried to encourage the discussion of light pollution in the media. A January 1996 front page article in the Sydney Morning Herald concentrated on the slow blotting out of the stars of that icon of the southern sky, the Southern Cross. This article caused considerable interest and led to many radio interviews. As a result of these protests, the illuminations of the building has been reduced by a slight reaiming 


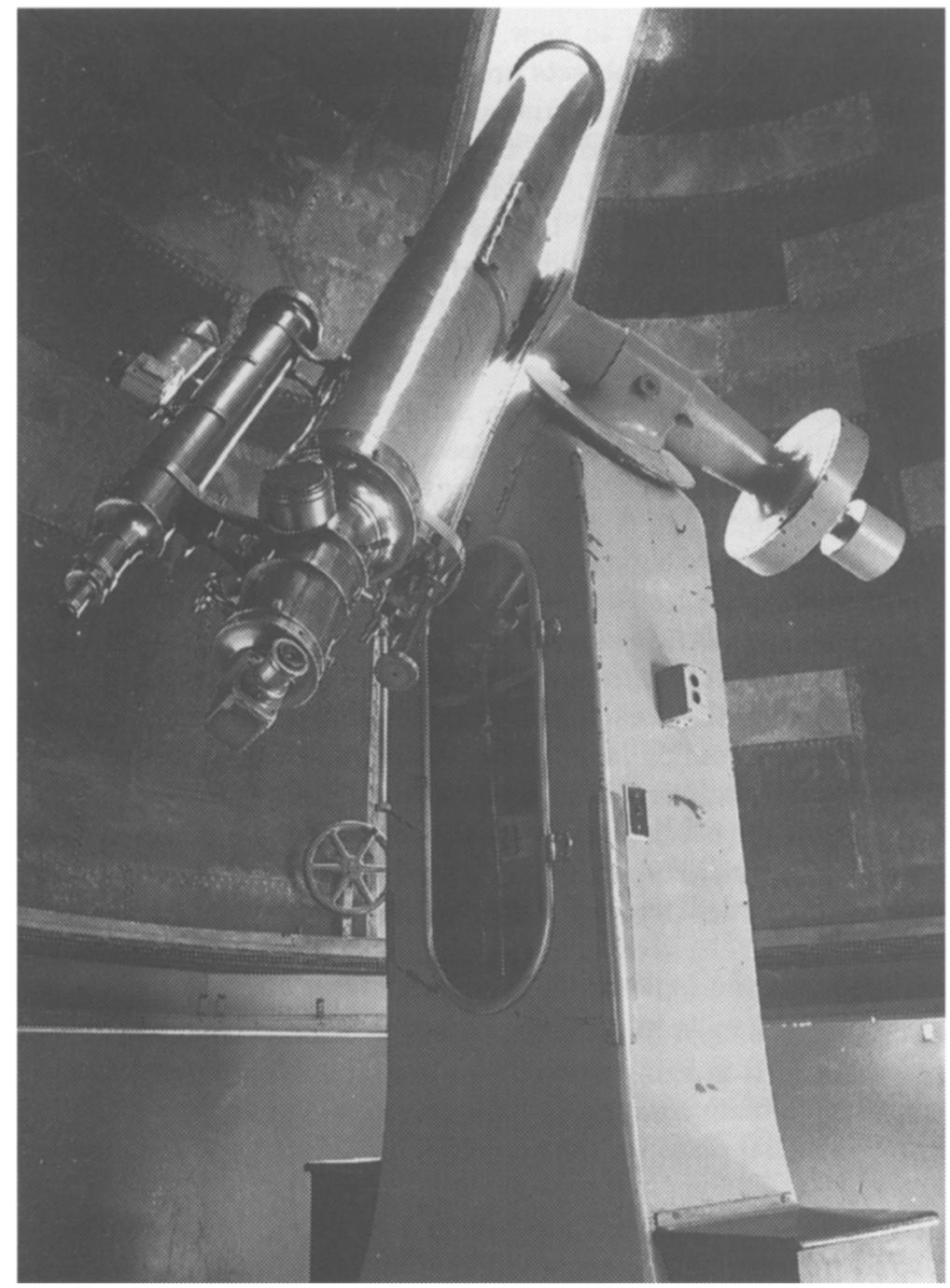

FIGURE 2. The historic $29 \mathrm{~cm}$ refracting telescope.

of the lights and council has imposed a curfew of $10 \mathrm{pm}$. Possibly, what is even more important is that the city council is now aware of the problem of light pollution.

\subsection{Telescopes}

There are two main telescopes at the observatory. In the south dome there is a historic 29 $\mathrm{cm}$ refractor dating from 1874 . In the other dome there is a computer controlled Meade LX200 16" $(40 \mathrm{~cm})$ Schmidt-Cassegrain telescope, which was installed in early 1995. The historic telescope is useful and provides an excellent way of demonstrating 19th century astronomy. However, the Meade has now become the preferred instrument for the public and the staff at the observatory.

The Meade is much easier to use as it is operated by push buttons instead of the physical manipulation necessary with the historic telescope. Its main advantage is its 
high pointing accuracy of a few minutes of arc. As a result any object aimed is within the field of view of the eyepiece. This overcomes the greatest difficulty at a bright sky site, which is to find the objects to view. If a telescope can be pointed in precisely the right direction, even quite faint objects are visible through it.

With the Meade telescope we are trialing the use of a CCD camera. A $500 \mathrm{~mm}$ focal length telescope has been installed on the main telescope and to this a CCD camera with small pixels has been attached. The field of view of the CCD camera approximately matches that through the eyepiece. In this way we can offer the public the direct look that they demand and yet show an image of the same object on a monitor. The image allows features of interest to be pointed out to visitors so that they know what to look for when they peer through the eyepiece.

\section{Exhibitions}

\subsection{Picturing the Sky: the Photographs of David Malin}

This is a small exhibition celebrating the work of the famous Australian astrophotographer, Dr David Malin. It comprises

- six large $(1.0 \times 1.2$ metre) colour photographic prints. Each print has a descriptive label and a sky map indicating the position of the object.

- a display case with the lens and a photographic plate from a pioneering study of the southern sky made from Sydney Observatory over a century ago. There are modern prints made by David Malin from the original negatives.

- The highlight of the exhibition is a touch screen computer interactive which allows visitors to take a journey in a spaceship to a variety of spectacular southern sky objects. At each object the recorded voice of Dr Malin provides expert guidance.

\subsection{By the Light of the Southern Stars: Planned for 1997}

By the Light of the Southern Stars is a major new exhibition that will utilise the whole observatory building. It is being planned for the second half of 1997. The exhibition is being developed by a team comprising of ourselves, designers and other colleagues from the museum. Our initial plans for the exhibition have just been accepted by the museum management.

Preparation of these initial plans has been a long and arduous process. The difficulty was trying to fit in many conflicting requirements into a building that has small rooms, little display space and that was designed as a working observatory and residence, not as a museum.

The current plan provides a satisfactory balance between historical and modern material. It begins with the astronomy of the Australian Aboriginal people whose stories about the Sun, the Moon and the stars reflect their belief in the spiritual unity of Nature and human beings (Haynes et al, 1996). It continues with an overview of Australian astronomy from James Cook, whose trip to observe the 1769 Transit of Venus led to European settlement, to the major instruments of the present day such as the Australia Telescope. Other historical sections include one on the main research achievements of Sydney Observatory and another discussing the relationship between time and navigation that led to the establishment of Sydney Observatory.

One of two largest rooms in the building will be targeted mainly at school groups. Through a mix of computer-based interactives, objects and working models it will cover aspects of the school syllabus such as the planets and the solar system, stars and constellations and the daily and yearly cycles of the sky. The other large room will be dedicated 
to modern astronomy and will emphasise the work and results from some of the main Australian research establishments.

\section{Discussion}

At Sydney Observatory we aim to interest and educate the public in astronomy. Currently we largely rely on our educational programs to do this as there are only a limited number of exhibits on display. They include the new Picturing the Sky exhibition (section 4.1) and remnants from the Hands on Astronomy exhibition discussed in Lomb and Wilson, 1990. The new educational initiatives such as the open nights and the camp-ins have been highly successful with the participation of many members of the public, both adults and children.

The most important activity at a public observatory is looking through a telescope. Thus it is essential to maintain and if possible to improve the facilities for viewing the sky. A major improvement has come with the addition of a large modern telescope and the provision of the capability of showing a CCD image. Our experience with the camera so far indicates that visitors do get more out of their look through the telescope when explanations are first made with a CCD image of the same object. However, it seems to make little difference whether the image is live or recorded from a previous occasion.

We are attempting to prevent further degradation of our night sky by fighting irresponsible attempts at light pollution. This is not only crucial for Sydney Observatory itself and amateur astronomers living in the city but in the long term for research astronomy as well. Sky glow from Sydney is already visible $400 \mathrm{~km}$ away at Siding Spring, Australia's premier site for optical astronomy (McNally, 1996).

Much work still has to be done in preparing our new exhibition for 1997 . We have to make sure that it is of interest and of relevance to our current and to potential visitors. It has to integrate with our educational and other activities. We are confident that $B y$ the Light of the Southern Stars will enhance the profile of the observatory and introduce many Australians and overseas visitors to astronomy and the southern sky.

\section{REFERENCES}

Haynes, R., Haynes, R., Malin, D. \& McGee, R., 1996, Explorers of the Southern Sky, Cambridge, Cambridge University Press.

Loмв, N.R., 1996, Publ. Astron. Soc. Aust. 13, 173-178.

Lomb, N.R. \& Wilson, T., 1990, The Teaching of Astronomy, Cambridge, Cambridge University Press, IAU Colloquium no. 105, 357-359.

MCNALLY, D., 1996, Q.J.R. astr. Soc. 37, 129-151. 